\title{
Joint LMMSE Estimation of DWI Data for DTI Processing
}

\author{
Antonio Tristán-Vega and Santiago Aja-Fernández \\ Laboratory of Image Processing \\ University of Valladolid. Spain \\ atriveg@lpi.tel.uva.es, sanaja@tel.uva.es \\ http://www.lpi.tel.uva.es
}

\begin{abstract}
We propose a new methodology for Linear Minimum Mean Square Error (LMMSE) filtering of Diffusion Weighted Imaging (DWI). We consider each voxel as an $N$-dimensional vector that comprises all the DWI volumes, and then compute the LMMSE estimator for the whole DWI data set jointly, taking into account the underlying tensor model. Our experiments, both with phantom and real data, show that this is a more convenient approach compared to the separate processing of each DWI, that translates to better removal of noise and preservation of structural information. Besides, our model has a simple algebraic formulation which makes the overall computational complexity very close to that of the scalar case, and it does not need multiple samples per DWI.
\end{abstract}

Keywords: LMMSE, DWI, DT-MRI, filtering.

\section{Introduction}

DWI allows the study of the diffusion direction of water molecules in the brain, which is associated in turn to the direction of fibre tracts in the white matter. It is well-known that DWI magnitude is lower for gradient directions near the diffusion direction due to the attenuation [1. Unlike structural Magnetic Resonance Imaging (MRI), where the signal to noise ratio (SNR) in usually large and the Rician nature of noise may be closely approximated with a Gaussian behaviour, the attenuation in DWI yields lower SNR's, so a Gaussian treatment of the signal introduces an important bias [2]. Typical approaches to DWI filtering include the conventional approach [3], based on the properties of the second order moment of Rician noise, Maximum Likelihood (ML) 4 and Expectation Maximisation (EM) 5] approaches, wavelets [6, or Wiener filtering [7]. Almost all of them take into account the bias that local averages introduce in the results due to the Rician nature of noise. In [8] the authors introduce the use of LMMSE estimation for the DWI squared magnitude, based on multiple samples: for each voxel and DWI volume, there is a vector of noisy samples, whose mean and covariance matrix are estimated to compute the LMMSE estimator. The simplicity and analytical tractability of this method make it very convenient. Its main limitation is that it requires multiple samples, which in general are not 
available. A different approach is proposed in [9] which no longer requires multiple samples; instead of a covariance matrix, the variance of each noisy magnitude is estimated, and therefore it is enough to have one single sample per DWI. The results shown in 9 demonstrate that the LMMSE performs better than a number of state of the art approaches. However, both methods process each DWI separately, dropping the important information related to the DWI formation mechanism. In this work our aim is to jointly process all DWI data: we have one single vector of samples at each image location that comprise all the DWI volumes, and compute the LMMSE estimator as a whole. The information given by the joint set of data is therefore more than the addition of the information given by each isolated DWI, since it includes information of the underlying physical model, so that the filtering results are more accurate than those of [8] and 9 .

\section{Joint LMMSE for DWI Data}

The model for the magnitude of each DWI signal is:

$$
M_{i}=\sqrt{\left(A_{i}+n_{c, i}\right)^{2}+n_{s, i}^{2}}
$$

where $M_{i}$ is the $i$-th noisy DWI component given by the MRI scanner, $n_{c, i}$ and $n_{s, i}$ are two independent zero-mean, white Gaussian processes, and $A_{i}$ is the ideal DWI magnitude in the absence of noise, given by the Stejskal-Tanner equation [1]:

$$
A_{i}=A_{0} \cdot \exp \left(-b \cdot g_{i}^{T} D g_{i}\right), i=1 \ldots N
$$

where $g_{i}$ is the unitary gradient direction, $D$ is the diffusion tensor (DT), $A_{0}$ is the baseline image value, and $b$ is a parameter of the scanner. Under the LMMSE suppositions, $\left\{A_{i}\right\}_{i=0}^{N}$ are considered as samples of random variables whose parameters are related to that of the observed values $\left\{M_{i}\right\}_{i=0}^{N}$. The oddorder moments of Rician distributed data like eq. (11) have no simple expressions, so it is common to work with the squared magnitude of the DWI values, since even-order moments have more tractable expressions. The LMMSE of $\mathbf{A}^{2} \equiv$ $\left[A_{0}^{2}, A_{1}^{2}, \ldots, A_{N}^{2}\right]^{T}, \widehat{\mathbf{A}^{2}}$, is therefore given for each voxel by [8]:

$$
\widehat{\mathbf{A}^{2}}=E\left\{\mathbf{A}^{2}\right\}+\mathbf{C}_{A^{2} M^{2}} \mathbf{C}_{M^{2} M^{2}}^{-1}\left(\mathbf{M}^{2}-E\left\{\mathbf{M}^{2}\right\}\right)
$$

where $\mathbf{C}_{A^{2} M^{2}}$ and $\mathbf{C}_{M^{2} M^{2}}$ are the cross-covariance and auto-covariance matrices. Meanwhile in [8] the components of $\mathbf{M}^{2}$ correspond to each consecutive sample of one single DWI (one vector per DWI), here each component corresponds instead to a DWI (one single vector for the whole DWI data set). Like in [8], the expected value $E\left\{\mathbf{M}^{2}\right\}$ is estimated as a local average in a neighbourhood of the voxel, $\left\langle\mathbf{M}^{2}\right\rangle$. It only remains to estimate the expected value $E\left\{\mathbf{A}^{2}\right\}$ and the covariance matrices $\mathbf{C}_{A^{2} M^{2}}$ and $\mathbf{C}_{M^{2} M^{2}}$; for the former, it is trivial to show from eq. (11) that assuming a noise variance $\sigma_{n}^{2}$ the following expression holds 1 :

$$
E\left\{\mathbf{A}^{2}\right\}=E\left\{\mathbf{M}^{2}\right\}-2 \sigma_{n}^{2} \simeq\left\langle\mathbf{M}^{2}\right\rangle-2 \sigma_{n}^{2}
$$

\footnotetext{
${ }^{1}$ Note that in an isotropic region, we simply have an average of the measured value $M_{i}^{2}$ to which we subtract the bias term $2 \sigma_{n}^{2}$, i.e. we construct an unbiased estimator.
} 
For the covariance matrix $\mathbf{C}_{M^{2} M^{2}}$, we simply use eq. (1)

$$
\begin{aligned}
\left\{\mathbf{C}_{M^{2} M^{2}}\right\}_{i, j}= & E\left\{\left(M_{i}^{2}-\overline{M_{i}^{2}}\right)\left(M_{j}^{2}-\overline{M_{j}^{2}}\right)\right\} \\
= & E\left\{\left(A_{i}^{2}+n_{c, i}^{2}+2 A_{i} n_{c, i}+n_{s, i}^{2}-\overline{A_{i}^{2}}-2 \sigma_{n}^{2}\right)\right. \\
& \left.\times\left(A_{j}^{2}+n_{c, j}^{2}+2 A_{j} n_{c, j}+n_{s, j}^{2}-\overline{A_{j}^{2}}-2 \sigma_{n}^{2}\right)\right\}
\end{aligned}
$$

where we have assumed the abbreviated notation $E\{X\} \equiv \bar{X}$. Taking into account the independence of the noise components with respect to the data and with respect to each other, we may develop eq. (5) to obtain:

$$
\begin{aligned}
\left\{\mathbf{C}_{M^{2} M^{2}}\right\}_{i, j}= & E\left\{\left(A_{i}^{2}-\overline{A_{i}^{2}}\right)\left(A_{j}^{2}-\overline{A_{j}^{2}}\right)\right\}+E\left\{2 A_{i} n_{c, i} \cdot 2 A_{j} n_{c, j}\right\} \\
& +E\left\{\left(n_{c, i}^{2}+n_{s, i}^{2}-2 \sigma_{n}^{2}\right)\left(n_{c, j}^{2}+n_{s, j}^{2}-2 \sigma_{n}^{2}\right)\right\} \\
= & \begin{cases}E\left\{\left(A_{i}^{2}-\overline{A_{i}^{2}}\right)\left(A_{j}^{2}-\overline{A_{j}^{2}}\right)\right\}, & i \neq j \\
E\left\{\left(A_{i}^{2}-\overline{A_{i}^{2}}\right)^{2}\right\}+4 \overline{A_{i}^{2}} \sigma_{n}^{2}+4 \sigma_{n}^{4}, & i=j\end{cases}
\end{aligned}
$$

since all other crossed terms are null. For covariance matrix $\mathbf{C}_{A^{2} M^{2}}$, a similar reasoning yields: $\left\{\mathbf{C}_{A^{2} M^{2}}\right\}_{i, j}=E\left\{\left(A_{i}^{2}-\overline{A_{i}^{2}}\right)\left(A_{j}^{2}-\overline{A_{j}^{2}}\right)\right\}$. The problem is that we have to estimate all crossed moments $E\left\{\left(A_{i}^{2}-\overline{A_{i}^{2}}\right)\left(A_{j}^{2}-\overline{A_{j}^{2}}\right)\right\}$, which translates in a high computational overload. Instead of doing so, we make the assumption that all expected values $\overline{A_{i}^{2}}$ are completely correlated, i.e., the value of each $\overline{A_{i}^{2}}$ univocally determines the values of all remaining $\overline{A_{j}^{2}}$. Since $\overline{A_{i}^{2}}$ are based on sample estimates of $\overline{M_{i}^{2}}$, this is equivalent to assuming that the variance of the estimate $\left\langle\mathbf{M}^{2}\right\rangle$ is small enough to assume that the inferred value of $\overline{\mathbf{A}^{2}}$ is close enough to the real value of $E\left\{\mathbf{A}^{2}\right\}$. With such an assumption, we have 2 :

$$
\begin{aligned}
& E\left\{\left(A_{i}^{2}-\overline{A_{i}^{2}}\right)\left(A_{j}^{2}-\overline{A_{j}^{2}}\right)\right\}=E\left\{A_{i}^{2} A_{j}^{2}\right\}-\overline{A_{i}^{2}} \overline{A_{j}^{2}} \\
= & \left(E\left\{A_{0}^{4}\right\}-\overline{A_{0}^{2}} \overline{A_{0}^{2}}\right) \exp \left(-b g_{i}^{T} D g_{i}\right) \exp \left(-b g_{j}^{T} D g_{j}\right) \\
= & \frac{\bar{A}_{0}^{4}-{\overline{A_{0}^{2}}}^{2}}{{\overline{A_{0}^{2}}}^{2}}{\overline{A_{0}^{2}}}^{2} \exp \left(-b g_{i}^{T} D g_{i}\right) \exp \left(-b g_{j}^{T} D g_{j}\right)=\frac{{\overline{A_{0}^{4}}}_{\bar{A}_{0}^{2}}{\overline{A_{0}^{2}}}^{2}}{\bar{A}_{i}^{2}} \overline{A_{j}^{2}}
\end{aligned}
$$

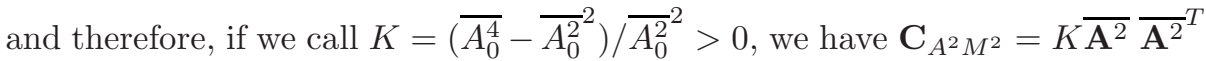
and $\mathbf{C}_{M^{2} M^{2}}=K \overline{\mathbf{A}^{2}}{\overline{\mathbf{A}^{2}}}^{T}+4 \sigma_{n}^{2} \operatorname{diag}\left(\overline{\mathbf{A}^{2}}\right)+4 \sigma_{n}^{2} I_{N+1}$, which only requires the estimation of the second order moments of all DWI components and the fourth order moment of the baseline image (not for all DWI's, like in [8]).

\footnotetext{
${ }^{2}$ Although we use here a simple tensor model, this reasoning remains valid for more general approaches, and it only requires that we assume the relation $A_{i}=A_{0} \cdot f\left(g_{i}\right)$.
} 


\section{Inversion of the Covariance Matrix of Measures}

The computation of covariance matrix $\mathbf{C}_{M^{2} M^{2}}$ (and respectively $\mathbf{C}_{A^{2} M^{2}}$ ) in eq. (6) is simple with the result in eq. (7), since we are able to estimate $\overline{A_{i}^{2}}$ from eq. (4), and an analogous expression is available for the fourth order moment: $\overline{A_{0}^{4}}=\overline{M_{0}^{4}}-8 \sigma_{n}^{2} \overline{A_{0}^{2}}-8 \sigma_{n}^{4}$. To estimate the noise power of $n_{c}$ and $n_{s}, \sigma_{n}^{2}$, several methods have been proposed (see [10]). The problem is that we need to invert $\mathbf{C}_{M^{2} M^{2}}$, which in general requires time-consuming Gaussian reduction. If we suppose that the noise power $\sigma_{n}^{2}$ is small related to the squared magnitude of the data, $\sigma_{n}^{2} \ll \overline{A_{i}^{2}}$, we may write:

$$
\mathbf{C}_{M^{2} M^{2}} \simeq \widetilde{\mathbf{C}}_{M^{2} M^{2}}=K \overline{\mathbf{A}^{2}}{\overline{\mathbf{A}^{2}}}^{T}+4 \sigma_{n}^{2} \operatorname{diag}\left(\overline{\mathbf{A}^{2}}\right) \Rightarrow \widetilde{\mathbf{C}}_{M^{2} M^{2}}^{-1}=\delta \overline{\overline{\mathbf{1}}}+\operatorname{diag}(\mathbf{e})
$$

where $\overline{\overline{\mathbf{1}}}$ is an $(N+1) \times(N+1)$ matrix with all elements equal to $1,\{\mathbf{e}\}_{i}=$ $\left(4 \sigma_{n}^{2} \cdot \overline{A_{i}^{2}}\right)^{-1}$ and $\delta=-\left(4 \sigma_{n}^{2}\left(4 \sigma_{n}^{2} / K+\sum_{i} \overline{A_{i}^{2}}\right)\right)^{-1}$. The proof of the right hand side of eq. (8) is straightforward, but we omit the details here for the sake of brevity. It is trivial as well to prove that $\widetilde{\mathbf{C}}_{M^{2} M^{2}}$ is always positive definite. Moreover, the eigenvalues $\left\{\lambda^{j}\right\}_{j=1}^{N+1}$ of $\widetilde{\mathbf{C}}_{M^{2} M^{2}}$ satisfy:

$$
\text { If } \overline{A_{i}^{2}}>\sigma_{n}^{2}, \forall i \quad \Rightarrow \quad \lambda^{j}>4 \sigma_{n}^{4}, \forall j
$$

Proof: Let us suppose that $\mathbf{v}^{j}$ is an eigenvector associated to eigenvalue $\lambda^{j}$; then: $\widetilde{\mathbf{C}}_{M^{2} M^{2}} \cdot \mathbf{v}^{j}=K \overline{\mathbf{A}^{2}}{\overline{\mathbf{A}^{2}}}^{T} \cdot \mathbf{v}^{j}+4 \sigma_{n}^{2} \operatorname{diag}\left(\overline{\mathbf{A}^{2}}\right) \cdot \mathbf{v}^{j}=\lambda^{j} \mathbf{v}^{j}$, and for the $i$-th component of $\mathbf{v}^{j}: K\left\langle\overline{\mathbf{A}^{2}}, \mathbf{v}^{j}\right\rangle \overline{A_{i}^{2}}+4 \sigma_{n}^{2} \overline{A_{i}^{2}} v_{i}^{j}=\lambda^{j} v_{i}^{j}$. For each non-null component of $\mathbf{v}^{j}$, we may write: $\left.\lambda^{j}=K \overline{\left\langle\mathbf{A}^{2}\right.}, \mathbf{v}^{j}\right\rangle \overline{A_{i}^{2}} / v_{i}^{j}+4 \sigma_{n}^{2} \overline{A_{i}^{2}}$. Now, if the projection of $\mathbf{v}^{j}$ in $\overline{\mathbf{A}^{2}}$ is positive, $\left\langle\overline{\mathbf{A}^{2}}, \mathbf{v}^{j}\right\rangle \geq 0$, since all $\overline{A_{i}^{2}}>0$ and $K>0$, there exists at least one positive component of $\mathbf{v}^{j}, v_{p}^{j}>0$, and therefore: $\lambda^{j}=K\left\langle\overline{\mathbf{A}^{2}}, \mathbf{v}^{j}\right\rangle \overline{A_{i}^{2}} / v_{p}^{j}+$ $4 \sigma_{n}^{2} \overline{A_{i}^{2}} \geq 4 \sigma_{n}^{2} \overline{A_{i}^{2}}>4 \sigma_{n}^{4}$. An analogous reasoning may be done if $\left\langle\overline{\mathbf{A}^{2}}, \mathbf{v}^{j}\right\rangle<0$.

The property in eq. (9) guarantees the convergence of the power series expansion of $\mathbf{C}_{M^{2} M^{2}}^{-1}$ as a function of $\widetilde{\mathbf{C}}_{M^{2} M^{2}}^{-1}$ :

$$
\begin{aligned}
& \mathbf{C}_{M^{2} M^{2}}^{-1}=\left(\widetilde{\mathbf{C}}_{M^{2} M^{2}}+4 \sigma_{n}^{4} I_{N+1}\right)^{-1}=\left(\widetilde{\mathbf{C}}_{M^{2} M^{2}}\left(I_{N+1}+4 \sigma_{n}^{4} \widetilde{\mathbf{C}}_{M^{2} M^{2}}^{-1}\right)\right)^{-1} \\
= & \left(I_{N+1}+4 \sigma_{n}^{4} \widetilde{\mathbf{C}}_{M^{2} M^{2}}^{-1}\right)^{-1} \widetilde{\mathbf{C}}_{M^{2} M^{2}}^{-1}=\widetilde{\mathbf{C}}_{M^{2} M^{2}} \sum_{k=0}^{\infty}\left(-4 \sigma_{n}^{4} \widetilde{\mathbf{C}}_{M^{2} M^{2}}^{-1}\right)^{k}
\end{aligned}
$$

where we have exploited the symmetry of covariance matrices to invert the order of the last two factors. We implement a truncated version of the series expansion $\sum_{k=0}^{\infty}\left(-4 \sigma_{n}^{4} \widetilde{\mathbf{C}}_{M^{2} M^{2}}^{-1}\right)^{k}\left(\mathbf{M}^{2}-\left\langle\mathbf{M}^{2}\right\rangle\right)$ with the following recursive rule:

$$
\mathbf{w}^{k+1}=\left(\mathbf{M}^{2}-\left\langle\mathbf{M}^{2}\right\rangle\right)-4 \sigma_{n}^{4} \widetilde{\mathbf{C}}_{M^{2} M^{2}}^{-1} \mathbf{w}^{k}, \quad \text { with } \mathbf{w}^{0}=\left(\mathbf{M}^{2}-\left\langle\mathbf{M}^{2}\right\rangle\right)
$$

It is trivial to show that the $k$-th element in the recursion of eq. (11) is the same as the $k$-th order approximation of the truncated series of eq. (10). 


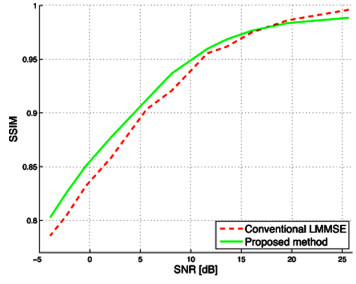

a

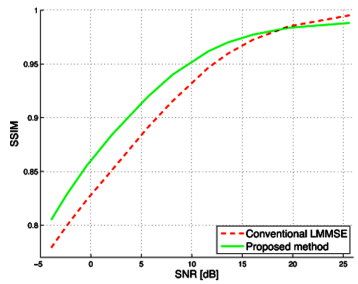

$\mathrm{d}$

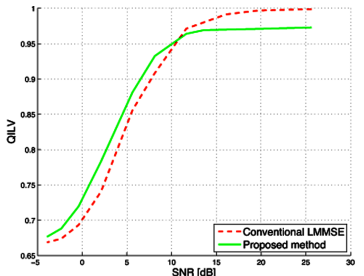

$\mathrm{b}$

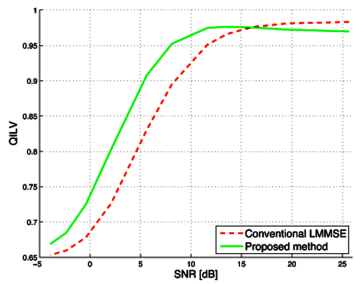

e

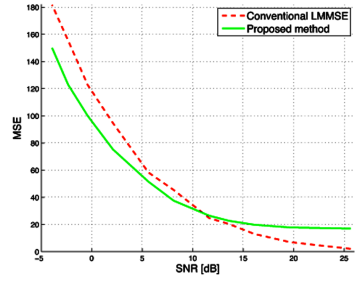

C

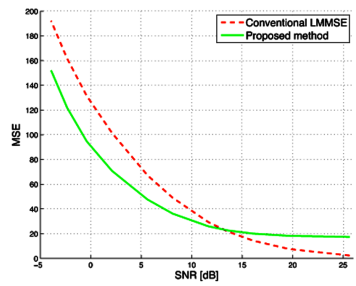

f

Fig. 1. SSIM (a, d), QILV (b, e) and MSE (c, f) as a function of the SNR. In the top row, 6 gradient directions have been used, meanwhile in the bottom row we use 27 . We compare our method to the case in which each DWI is separately filtered, like in [8].

We can apply the procedure described in eqs. (8) and (11) for all those image voxels satisfying eq. (9). If this last condition is not fulfilled, an explicit Gaussian elimination is required to invert $\mathbf{C}_{M^{2} M^{2}}$; fortunately, this happens for a negligible percentage of image voxels. The products with $\widetilde{\mathbf{C}}_{M^{2} M^{2}}^{-1}$ are computationally very efficient, since we do not need to perform explicit matrix products, but instead we have: $\left\{\widetilde{\mathbf{C}}_{M^{2} M^{2}}^{-1} \cdot \mathbf{w}\right\}_{i}=\delta \sum_{j} w_{j}+\{\mathbf{e}\}_{i} \cdot w_{i}$. On the other hand, we have that $\mathbf{C}_{A^{2} M^{2}} \cdot \mathbf{w}=K\left\langle\overline{\mathbf{A}^{2}}, \mathbf{w}\right\rangle \overline{\mathbf{A}^{2}}$, so the computation of eq. (3) may be done very efficiently, and the overall computational complexity remains low.

\section{Results}

To test our method, we have compared it to that of [9] for two main reasons: first, the LMMSE approach in [9] has been proved to outperform a number of filtering techniques in the more recent state of the art, and second the formulation in [9] is equivalent to that of [8] in case only one sample per DWI is available, these two being the only LMMSE approaches to DWI filtering in the literature to the very best of our knowledge.

We have designed a synthetic $256 \times 256 \times 81$ (resolution $1 \times 1 \times 256 / 81$ ) tensor field as a spherical object of radius $R=120$ inserted in a black background. The baseline image has a decreasing magnitude from the centre to the borders: $A_{0}=230 /\left(1+(r / 200)^{2}\right)$; a band with constant magnitude $A_{0}=255$ has been inserted for $|x|<35$. Three anisotropic strips have been designed along each coordinate axis, with diffusion directions following these axis: for $|y, z|<35$, we 


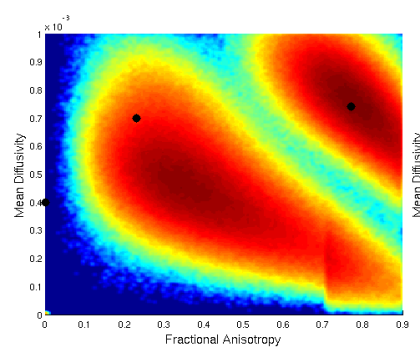

a

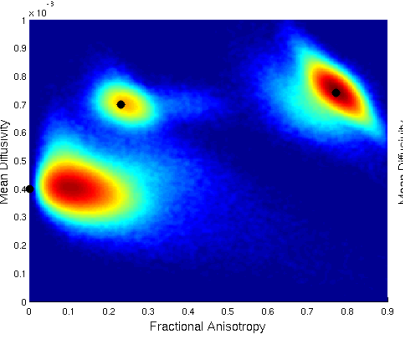

$\mathrm{b}$

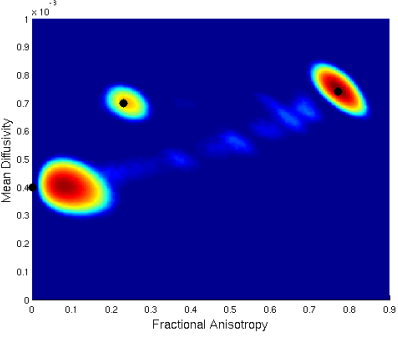

C

Fig. 2. Log-plots of the $2 \mathrm{D}$ histogram of the pairs (FA,MD), for (a) the noisy data, (b) the data filtered with the approach in 819, and (c) our own approach, with $\mathrm{SNR}=12 \mathrm{~dB}$ and 27 gradient directions. Ground truth centroids are represented as black spots, representing the isotropic zone $\left(0,0.4 \cdot 10^{-3}\right)$, the anisotropic strips $\left(0.77,0.73 \cdot 10^{-3}\right)$, and the crossing zone $\left(0.23,0.7 \cdot 10^{-3}\right)$.

have eigenvalues $\lambda=\left[1 \cdot 10^{-3}, 0.2 \cdot 10^{-3}, 0.2 \cdot 10^{-3}\right]$, and so on. The rest of the phantom is assumed to be an isotropic medium with $\lambda^{j}=0.25 \cdot 10^{-3}, \forall j$. The $b$-value of the scanner has been fixed to 1200 . We generate two sets of data using eq. (2), with 6 and 27 gradient directions $(N=7$ and $N=28$, respectively) uniformly distributed in the unit sphere. We add Rician distributed noise with the model in eq. (1), varying the noise power $\sigma_{n}^{2}$. To compute the SNR, the signal power is measured as [9]: $S=\min _{i, x, y} A_{i}(x, y)$, which gives us a range of $-5 d B \leq S N R \leq 25 d B$, i.e., a noise power $3.5 \leq \sigma_{n} \leq 100$, much like in 9 . We have used a window of $5 \times 5 \times 1$ voxels to estimate local averages.

Like in [8], we use the Structural Similarity (SSIM) index [11] and the Quality Index based on Local Variance (QILV) [12] as performance measures, together with the Mean Square Error (MSE). We remove the background from the computation to avoid any bias in the results. The results may be seen in Fig. 1. Our method outperforms the techniques based on the separate filtering of each DWI for reasonable degrees of noise, which is, SNR below 10dB for 6 gradients or below $15 \mathrm{~dB}$ for 27 gradients. It is worth notice that the difference between our approach and those of [89] grows as the number of gradients increase, since more joint information is available. To test the potential of our method in DTI processing, we have estimated the DT both from the noisy and the filtered data by means of a Weighted Least Squares (WLS) fitting [2], and then we have computed the Mean Diffusivity (MD) and the Fractional Anisotropy (FA). Log-plots of the corresponding histograms for $\mathrm{SNR}=12 \mathrm{~dB}$ and 27 gradient directions may be seen in Fig. 2. Our approach yields more accurate results, showing more compact clusters around the centroid\$3. As a quality measure, we give the mean euclidean distance of the pair (FA,MD) to its corresponding ground-truth centroid (we ponder MD with a factor of 1000): for the noisy data we have $\mathbf{0 . 3 4}$,

${ }^{3}$ The centroid with $\mathrm{FA}=0$ is not well accounted in any case; this is because $\mathrm{FA}=0$ means that all eigenvalues are identical, which is impossible in practise with noisy data; however, note that the values of FA are nearly 0 for this group. 


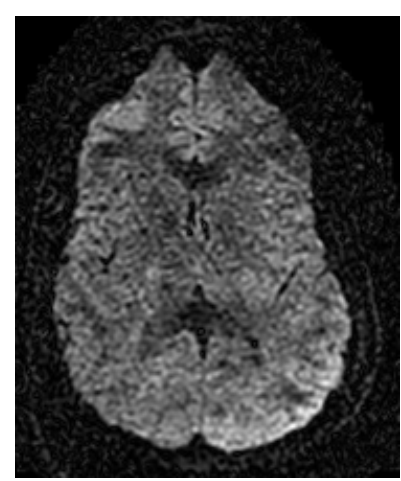

a

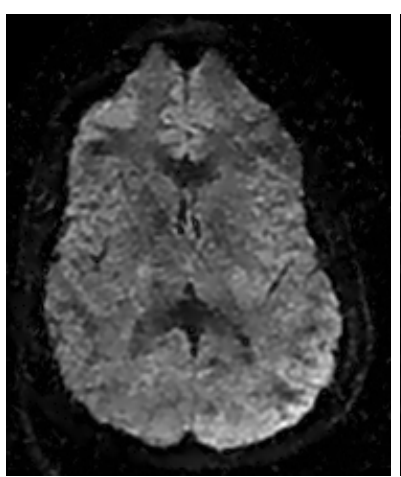

$\mathrm{b}$

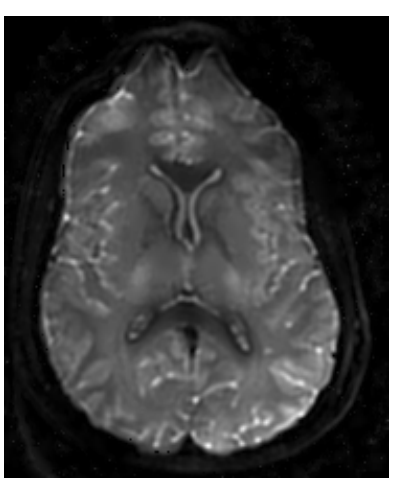

C

Fig. 3. An experiment with real data: we have a $184 \times 221 \times 78$ volume with 51 gradient directions; we represent the original axial slice of the first gradient direction (a), and the results for the approach in 8]9] (b) and our own approach (c).

for the approach in $89, \mathbf{0 . 1 0}$, and for our own approach, 0.08. Regarding computation times, both approaches take roughly 4.5 seconds to execute in our $2 \mathrm{GHz}$., 8-core, 32GB machine, with 27 gradient directions., i.e. our algorithm needs no extra computation time compared to [9].

Finally, we present in Fig. 3 an experiment with a real SENSE EPI data set4, scanned in a 3 Tesla GE system, with 51 gradient directions, 8 baselines, and resolution $0.9375 \times 0.9375 \times 1.7 \mathrm{~mm}^{3}$. Our algorithm is able to recover structures which remain hindered in both the original gradient image and in the LMMSE filtered, due to the combined use of all DWI data for each image location and gradient. Note especially the high improvement in the surroundings of the ventricles.

\section{Conclusion}

We have proposed a new methodology for LMMSE estimation of DWI data, based on the use of the joint information of the whole DWI data set. Compared to [89], the experimental results show that our approach yields a better removal of noise while better preserving the structural information. Like these previous works, our filter is unbiased, which is an important property when estimating the DT [2]. LMMSE has been proved to outperform the most important approaches in the related literature [9], such as the conventional approach [3, ML [4] or EM [5] amongst others, with little computational load. Our approach outperforms that of [9] with no additional computational cost. This is mainly because we do not need to explicitly estimate covariance matrices, and we may use analytical expressions to invert them. This makes our algorithm very efficient compared to [8], and we no longer need multiple samples per DWI. One last advantage is that

\footnotetext{
${ }^{4}$ Courtesy of PNL, Dept. of Psychiatry, and LMI Dept. Radiology, Brigham and
} Women's Hospital, Boston. 
our method requires the computation of the fourth order moment only for the baseline image, which is always the one with the higher SNR, so the uncertainty in the computation of this value may be reduced.

Acknowledgments. This work was partially supported by grant number TEC2007-67073/TCM from the Comisión Interministerial de Ciencia y Tecnología, Spain.

\section{References}

1. Stejskal, E.O., Tanner, J.E.: Spin diffusion measurements: Spin echoes in the presence of a time-dependent field gradient. J. of Chem. Phys. 42, 288-292 (1965)

2. Salvador, R., Pea, A., Menon, D.K., Carpenter, T.A., Pickard, J.D., Bullmore, E.T.: Formal characterization and extension of the linearized diffusion tensor model. Hum. Brain Mapp. 24, 144-155 (2005)

3. McGibney, G., Smith, M.: Unbiased signal to noise ratio measure for magentic resonance images. Med. Phys. 20(4), 1077-1078 (1993)

4. Sijbers, J., den Dekker, A.J.: Maximum Likelihood estimation of signal amplitude and noise variance from MR data. Magn. Res. Imag. 51, 586-594 (2004)

5. Marzetta, T.: EM algorithm for estimating the parameters of multivariate complex Rician density for polarimetric SAR. In: Procs. of ICASSP, vol. 5, pp. 3651-3654 (1995)

6. Nowak, R.: Wavelet-based Rician noise removal for Magnetic Resonance Imaging. IEEE Trans. on Im. Proc. 8(10), 108-1419 (1999)

7. Martín-Fernández, M., Alberola-López, C., Ruiz-Alzola, J., Westin, C.F.: Sequential anisotropic Wiener filtering applied to 3D MRI data. Magn. Res. Imag. 25, 278-292 (2007)

8. Aja-Fernández, S., Alberola-López, C., Westin, C.F.: Signal LMMSE estimation from multiple samples in MRI and DT-MRI. In: Ayache, N., Ourselin, S., Maeder, A. (eds.) MICCAI 2007, Part II. LNCS, vol. 4792, pp. 368-375. Springer, Heidelberg (2007)

9. Aja-Fernández, S., Niethammer, M., Kubicki, M., Shenton, M.E., Westin, C.F.: Restoration of DWI data using a Rician LMMSE estimator. IEEE Trans. on Med. Imag (in press, 2008)

10. Sijbers, J., den Dekker, A.J., Van Audekerke, J., Verhoye, M., Van Dyck, D.: Estimation of the noise in magnitude MR images. Magn. Res. Imag. 16(1), 87-90 (1998)

11. Wang, Z., Bovik, A.C., Sheikh, H.R., Simoncelli, E.P.: Image quality assessment: form error visibility to structural similarity. IEEE Trans. on Im. Proc. 13(4), 600612 (2004)

12. Aja-Fernández, S., San-José-Estépar, R., Alberola-López, C., Westin, C.F.: Image quality assessment based on local variance. In: Procs. of IEEE EMBC, New York, USA, pp. 4815-4818 (2005) 